\title{
NON-LINEAR SELF-ACCELERATION OF ELECTRONS EMITTED BY PLASMA CATHODE
}

\author{
A.V. Pashchenko, I.A. Pashchenko
}

\author{
National Science Center "Kharkov Institute of Physics and Technology”, Kharkov, Ukraine \\ e-mail: paschenko@kipt.kharkov.ua
}

A non-linear non-stationary analytical theory of diode gap overlap by electrons emitted from the plasma cathode is represented. The fact of the non-linear self-acceleration at the electron flow front is confirmed.

PACS: 52.25.Sw, 52.35.Py, 52.60+h

\section{INTRODUCTION}

Electron self-acceleration takes place in a cathodeanode interval during electron emission from a metal cathode [1]. This effect provides the appearance of the corresponding additional source of current in the equivalent electrical circuit of the diode. It is interesting to ascertain as this effect is modified in case of electron emission from a plasma cathode. This problem is investigated in the present report.

\section{THEORY}

Let us consider a plasma layer with a thickness of $d$ in the Cartesian coordinate system $0 X Y Z$ placed between two conducting plates $x=0$ and $x=l$ in the interval $(0, d)$, i.e. $d<1$.

Since a moment $t=0$ the plates $x=0$ and $x=l$ were found under potentials $\varphi(0)=0$ and $\varphi(l)=\varphi_{0}(t)>0$, respectively. So at $t>0$ an external electric field acts on plasma. Then to a moment $t$ not so greater than $t=0$ electrons are displaced from the initial position so that the whole region $(0, l)$ is divided on four physical regions: a) $(0, k)$ - ions; b) $(k, d)$ - plasma; c) $(d, m)$ electrons; d) $(m, l)$ - vacuum. Let us consider an electrodynamical problem for each of these regions with taking into account the non-linear electron movement, make a joining of fields and potentials and satisfy their boundary conditions.

Let us start to proceed from the electron movement equation, continuity equation and Poisson equation which in the Lagrange variables $a, t$, where $a$ is the initial coordinate, $t$ is the time, have a form:

$$
\begin{aligned}
& \partial v / \partial t=-e / m E, \\
& n \partial x / \partial a=C_{1}(a), \\
& \partial E / \partial a=4 \pi e\left(n_{i}-n\right) \partial x / \partial a, \\
& n_{i}=n_{i}(a),
\end{aligned}
$$

where $e$ and $m$ are the charge value and the mass of electron.

Let us consider that at $t=0$ and $x=a: v=0, n_{i}(a)=n_{0}$. Then $C_{1}(a)=n_{0}$ and from equation (3) we have

$$
E=4 \pi e n_{0}(x-a)+\xi(t),
$$

\section{We have from equation (1):}

$$
x=a+\theta(t),
$$

where function $\theta(t)$ satisfies the equation

$$
\theta_{t^{2}}^{\prime \prime}+\omega{ }_{p}^{2} \theta+e / m \xi(t)=0
$$

and besides $\left.\theta\right|_{t=0}=0,\left.\theta^{\prime}\right|_{t=0}=0$.

Then we found that in this region

$$
\varphi=m / e^{\left[\theta "(x-\theta)+H_{1}(t)\right],}
$$

where $H_{l}(t)$ is the integral constant.

In this region the electron movement is described by the set of equations

$$
\left\{\begin{array}{l}
\partial v / \partial t=-e / m E \\
n \partial x / \partial \tau=C_{2}(\tau), \\
\partial E / \partial x=-4 \pi e n
\end{array}\right.
$$

where $\tau$ is the time of passing by an electron of the coordinate $x=d$.

The integration of the Poisson equation from (9) has shown that the electric field represents a sum of function $t$ and function $\tau$ in this region:

$$
E=-4 \pi e \int_{0}^{\tau} C_{2}(\xi) d \xi+C_{3}(t) .
$$

It permits to execute the integration of movement equation in quadratures:

$$
\begin{aligned}
& \partial^{2} x / \partial t^{2}=-e / m_{i}\left[-4 \pi e \int_{0}^{\tau} C_{2}(\xi) d \xi+C_{3}(t)\right], \\
& \partial x / \partial \mathrm{t}=\omega^{2} / n_{0} /\left(\int_{0}^{\tau^{\prime}} C_{2}(\xi) d \xi\right)(t-\tau)- \\
& -\frac{e}{m} \int_{\tau}^{t} C_{3}(\xi) d \xi+C_{4}(\tau), \\
& x=C_{5}(\tau)+{ }^{\omega}{ }_{p}^{2} / n_{0}\left(\int_{0}^{\tau} C_{2}(\xi) d \xi\right)(t-\tau)^{2} / 2- \\
& -e / m \int_{\tau}^{t} d \xi_{1} \int_{\tau}^{\xi_{1}} C_{3}(\xi) d \xi+C_{4}(\tau)(t-\tau) .
\end{aligned}
$$

We find the integration constants from conditions at $t=\tau$.

$$
\begin{aligned}
& C_{5}(\tau)=\left.x(\tau, t)\right|_{t=\tau}=d, \\
& C_{4}(\tau)=\left.\frac{\partial x(\tau, t)}{\partial t}\right|_{t=\tau}=\left.v\right|_{t=\tau}=\left.\frac{\partial x(a, t)}{\partial t}\right|_{x=d}=\frac{d \theta(\tau)}{d \tau} .
\end{aligned}
$$

where $\xi(t)$ is the integral constant. 
Now we can find the function $C_{2}(\tau)$ incoming into the continuity equation in (9):

$$
C_{2}(\tau)=n \partial x / \partial \tau=\left.n\right|_{t=\tau} \partial x /\left.\partial \tau\right|_{t=\tau}=\left.n\right|_{x=d} \partial x /\left.\partial \tau\right|_{t=\tau} .
$$

Since $\left.n\right|_{x=d}=n_{0}$ and $\partial x /\left.\partial \tau\right|_{t=\tau}=-C_{4}(\tau)+d C_{5}(\tau) / d \tau=-\theta_{\tau}^{\prime}(\tau)$,

$$
C_{2}(\tau)=-n_{0} \theta_{\tau}^{\prime}(\tau) \text {. }
$$

Joining of electric fields at the point $x=d$ leads to the formula

$$
C_{3}(t)=-m / e^{\theta \prime}(t)-4 \pi e n_{0} \theta(t) .
$$

Then taking into account that

$$
\partial x / \partial t=-\theta^{\prime}(\tau)\left[1+\omega_{p}^{2}(t-\tau)^{2} / 2\right],
$$

we can find the potential in the region $(d, m)$

$$
n(\tau, t)=\left(2 n_{0}\right) /\left(2+\omega_{p}^{2}(t-\tau)^{2}\right) .
$$

It can be seen from formula (18) that the electron density decreases with time as we move off from the plasma layer boundary $x=d$.

The second boundary coordinate of the plasma layer is determined by the formula, getting from (13):

$$
m=x(0, t)=d+\theta(t)+\omega{ }_{p}^{2} \int_{0}^{t} d \xi_{1} \int_{0}^{\xi_{1}} \theta(\xi) d \xi .
$$

It can be seen that the electron coordinate changing at layer boundary differs from the corresponding change in the plasma region at the value $\omega_{p}^{2} \int_{0}^{t} d \xi_{1} \int_{0}^{\xi_{1}} \theta(\xi) d \xi$ that confirms the electron layer propagation.

Consideration of the Poisson equation $\partial E_{3} / \partial x=0$, joining of fields and potentials in the point $x=m$, the connection to boundary condition on the border $x=l$ leads to the formulas:

$$
\begin{aligned}
& E_{3}(x, t)=-(m / e)\left(\theta^{\prime \prime}+\omega{ }_{p}^{2} \theta\right), \\
& \varphi_{3}(x, t)=-(m / e)\left(\theta^{\prime \prime}+\omega{ }_{p}^{2} \theta\right)(l-x)+\varphi_{0}(t), \\
& H_{1}(t)=(e / m) \varphi_{0}(t)-\theta^{\prime \prime}(l-\theta)-\omega{ }_{p}^{2} \theta(l-d)+ \\
& +\omega{ }_{p}^{2} \int_{0}^{t} \theta(\xi) \theta^{\prime}(\xi)\left[1+(t-\xi)^{2} / 2\right] d \xi
\end{aligned}
$$

where $\varphi_{0}(t)$ is the potential of anode $x=l$.

A positively charged layer completed by heavy stationary ions is appeared near the cathode after beginning of action of the external potential $\varphi_{0}(t)$. The ion density is $n_{0}$. Solving of the Poisson equation $\partial E_{1} / \partial$ $x=4 \pi e n_{0}$ and joining of electrical fields and potentials in the point $x=k$ give us:

$$
\begin{aligned}
& E_{1}=4 \pi e n_{0} x+\xi(t), \\
& \varphi_{1}=-2 \pi e n_{0} x^{2}-\xi(t) x, \\
& H_{1}(t)=\theta \theta^{\prime \prime}+\omega_{p}^{2} \theta^{2} / 2 .
\end{aligned}
$$

\section{BASIC PROBLEM EQUATION AND RESULTS}

Equating expressions (22) and (25), we receive the equation for determination of non-linear electron displacement in the plasma layer $\theta$ :

$$
\frac{d^{2} \rho}{d T^{2}}+(1-\eta) \rho-\phi_{0}(T)-\int_{0}^{T} \rho(\xi) \rho^{\prime}(\xi) \frac{(T-\xi)^{2}}{2} d \xi=0,
$$

where $\rho \equiv \theta / l, T \equiv \omega_{p} t, \eta \equiv d / l, \phi_{0}(T) \equiv\left(e \varphi_{0}(t)\right) /\left(m \omega{ }_{p}^{2} l^{2}\right)$.
The equation (26) is the head result of present research. The numerical solution of this equation was performed using the function

$$
\phi_{0}(T)=\gamma\left(1-e^{-\alpha T}\right) \text {. }
$$

The concrete values of parameters $\eta$ and $\gamma$ are very important for result. Estimations give us the value $10^{-3}$ for $\gamma$ under $l \approx 1 \mathrm{sm}, n_{0}=10^{12} \mathrm{sm}^{-3}$ and potential amplitude $\varphi_{0}(t) \mid \approx 10^{3} \mathrm{~V}$. The level of effect on plasma can be characterized by the ratio $\rho / \eta$ : the effect is strong for $\rho /$ $\eta \leq 1$, and the effect is low for $\rho / \eta<<1$.

Fig. 1-3 demonstrate the numerical results for $\gamma$ $=0,025, \eta=0,5$. In particular, Fig. 2 shows the velocity of one electron corresponding. This fact takes place due to the collective acceleration of electrons at the electron flow front.

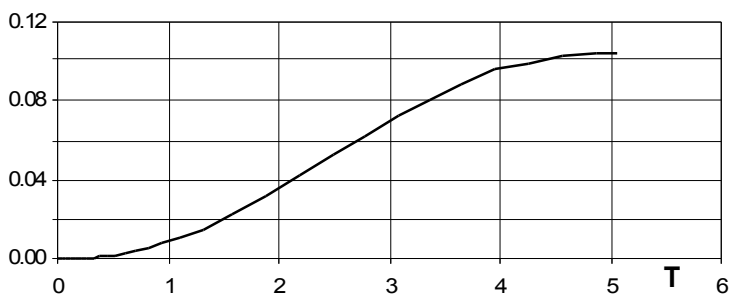

Fig. 1. Electron displacement in a plasma layer, $\rho$.

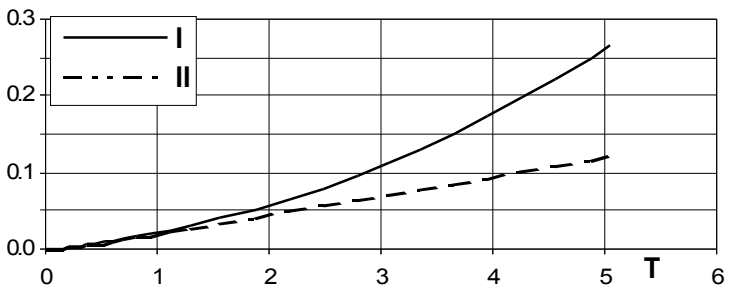

Fig. 2. Curve I - velocity of electron flow front, curve II - velocity of one electron under the influence of $\Phi_{0}(T)$ for the case $a=d$

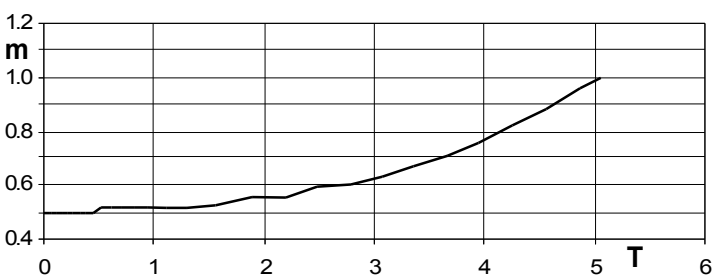

Fig. 3. The coordinate of electron flow front

\section{CONCLUSION}

In this report we create a strong non-linear nonstationary analytical theory of diode gap overlap by electrons emitted from the plasma cathode. The fact of the non-linear self-acceleration at the electron flow front is confirmed.

\section{REFERENCES}

1. A.V. Pashchenko, V.E. Novikov, Y.V. Tkach. The phenomenon of non-linear cathode-emitted particle self acceleration. 10th IEEE International Pulsed Power Conference, Albuquerque, New Mexico, 1995, p. 852-856. 